\title{
Anti-PCSK9 antibodies for the treatment of heterozygous familial hypercholesterolemia: patient selection and perspectives
}

REVIEW

This article was published in the following Dove Press journal:

Vascular Health and Risk Management

4 September 2017

Number of times this article has been viewed

\author{
Alberico Luigi Catapano ${ }^{1,2}$ \\ Angela Pirillo',2 \\ Giuseppe Danilo Noratal,3,4 \\ 'Department of Pharmacological and \\ Biomolecular Sciences, Università \\ degli Studi di Milano, ${ }^{2}$ IRCCS \\ Multimedica Hospital, Sesto San \\ Giovanni, ${ }^{3}$ Center for the Study of \\ Atherosclerosis, E. Bassini Hospital, \\ Cinisello Balsamo, Milan, Italy; ${ }^{4}$ School \\ of Biomedical Sciences, Curtin Health \\ Innovation Research Institute, Curtin \\ University, Perth, WA, Australia
}

Correspondence: Giuseppe Danilo Norata

Department of Pharmacological and Biomolecular Sciences - Università degli Studi di Milano, Via Balzaretti 9, 20I33,

Milan, Italy

$\mathrm{Tel}+390250318313$

$\mathrm{Fax}+390250318386$

Email danilo.norata@unimi.it

\begin{abstract}
Heterozygous familial hypercholesterolemia $(\mathrm{FH})$ is a genetic disorder characterized by high low-density lipoprotein cholesterol levels from birth, which exposes the arteries to high levels of atherogenic lipoproteins lifelong and results in a significantly increased risk of premature cardiovascular events. The diagnosis of $\mathrm{FH}$, followed by an appropriate and early treatment is critical to reduce the cardiovascular burden in this population. Phase I-III clinical trials showed the benefit of proprotein convertase subtilisin kexin 9 inhibitors, both alirocumab and evolocumab, in these patients with an average low-density lipoprotein cholesterol reduction ranging from $-40 \%$ to $-60 \%$. The aim of this review is to address the unmet needs in cholesterol management, elucidate the biology and the clinical benefit of proprotein convertase subtilisin kexin 9 inhibition and finally discuss the open gaps and future directions in the treatment of patients with heterozygous FH.
\end{abstract}

Keywords: HeFH, dyslipidemia, cholesterol, alirocumab, evolocumab

\section{Unmet needs in cholesterol management: focus on heterozygous familial hypercholesterolemia}

Heterozygous familial hypercholesterolemia $(\mathrm{HeFH})$ is a genetic disorder characterized by high low-density lipoprotein cholesterol (LDL-C) levels from birth, which exposes the arteries to high levels of atherogenic lipoproteins lifelong and results in a significantly increased risk of premature cardiovascular events. ${ }^{1}$ The diagnosis of familial hypercholesterolemia (FH), followed by appropriate and early treatments aimed at reducing LDL-C levels are therefore critical to reduce the cardiovascular burden in this population.

$\mathrm{FH}$ is caused primarily by mutations in the gene encoding for the LDL receptor (LDLR) which, by affecting its structure and function, cause decreased removal of LDL from the circulation. Mutations in the $L D L R$ gene account for $\sim 90 \%$ of genetically determined $\mathrm{HeFH}$, the remaining is due to mutations which alter the binding site of apoB to the LDLR, or to other mutations increasing the activity of proprotein convertase subtilisin kexin 9 (PCSK9), which cause a decrease in LDLR expression/ activity. ${ }^{2}$ Heterozygous subjects present with elevated plasma LDL-C levels (200-500 $\mathrm{mg} / \mathrm{dL})$. The frequency of HeFH in the general population has been estimated in $1: 200-250,{ }^{3}$ and is higher in selected populations such as patients with premature cardiovascular disease. ${ }^{4}$ Despite that, $\mathrm{HeFH}$ is still underdiagnosed and, as a consequence, undertreated. The homozygous form of FH is much rarer $(1: 160,000-300,000) .{ }^{5}$ These patients present with very high LDL-C levels (untreated levels $>500 \mathrm{mg} / \mathrm{dL}$ ) and are at extremely elevated risk of cardiovascular events. ${ }^{5}$ 
The diagnosis of FH can be done relatively easily by using clinical tools such as the Dutch Lipid Clinic Network (DLCN) criteria, ${ }^{6}$ the Make Early Diagnosis to Prevent Early Death (MEDPED) criteria, ${ }^{7}$ or the Simon Broome (SB) criteria (Table 1). ${ }^{8}$ Depending on the specific criteria, the score is calculated based on the presence of high LDL-C levels, on patient history of premature coronary heart disease (CHD) or cerebral or peripheral vascular disease, on family history of premature CHD or hypercholesterolemia and on the presence of physical signs such as tendon xanthomas or corneal arcus. The MEDPED criteria rely on age-specific and family relative-specific levels of total cholesterol, but do not integrate this information with the clinical characteristics of the subjects or the identification of a FH mutation. The DLCN score takes into account a family or personal history of premature CHD, physical signs, and high LDL-C levels, and suggests the genetic analysis if the score is $>5$; a definite $\mathrm{FH}$ diagnosis is given when the score is $>8$. SB criteria are similar in terms of parameters evaluated for the score calculation, giving a definite $\mathrm{FH}$ diagnosis in the presence of high

Table I Clinical criteria for the diagnosis of familial hypercholesterolemia

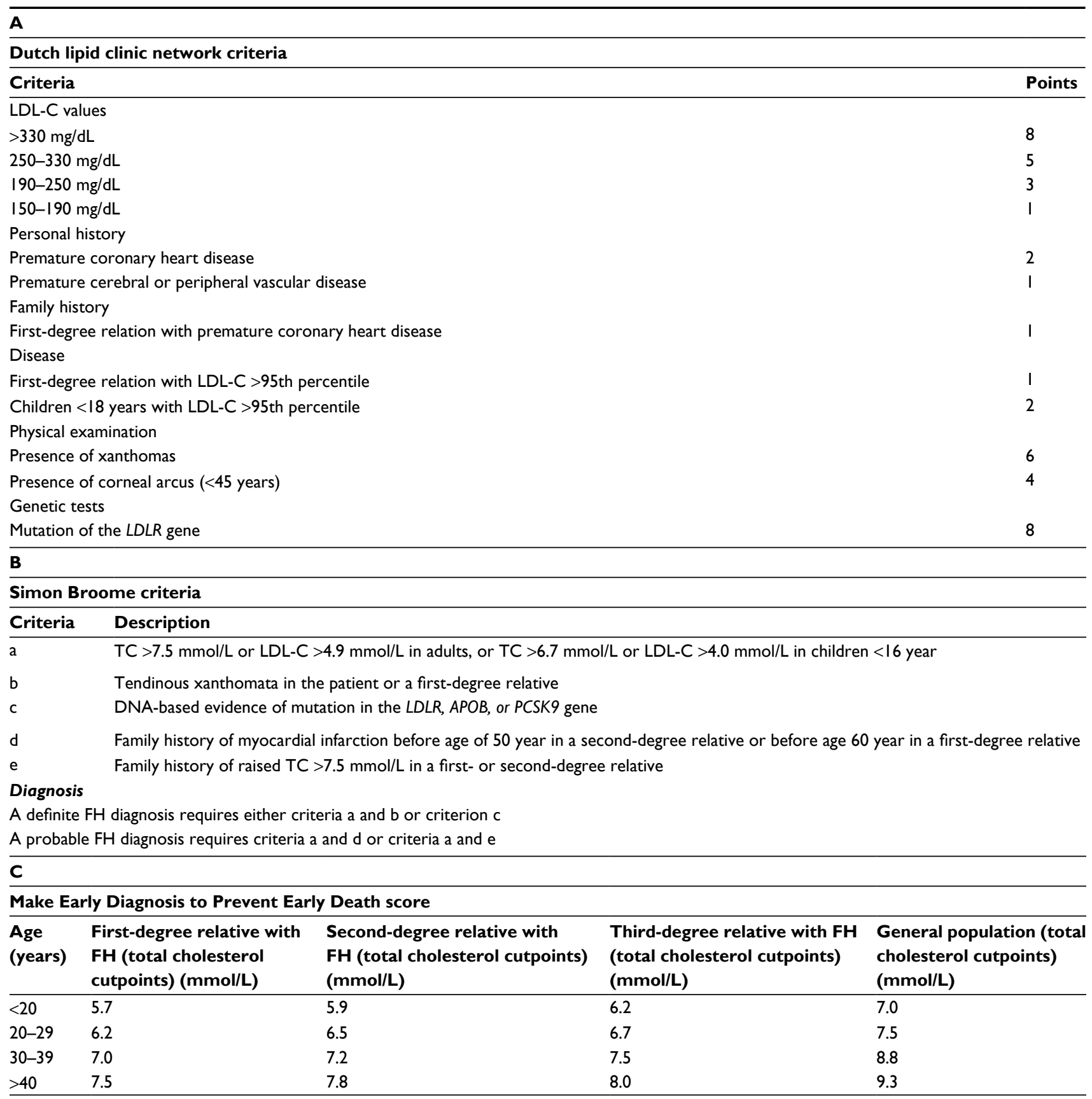

Note: Diagnosis: FH is diagnosed if total cholesterol levels exceed the cut point.

Abbreviations: FH, familial hypercholesterolemia; LDL-C, low-density lipoprotein cholesterol; TC, total cholesterol. 
LDL-C (or total cholesterol) levels plus tendon xanthomas in the patient or a first or second-degree relative or in the presence of a functional mutation in one of the 3 candidate genes (Table 1).

Current guidelines suggest to lower LDL-C as early as possible in $\mathrm{HeFH}$ patients, to delay the onset of the first cardiovascular event. ${ }^{3,9}$ Recommended LDL-C goals for these patients are levels $<100 \mathrm{mg} / \mathrm{dL}(<2.5 \mathrm{mmol} / \mathrm{L})$ for adults, or $<70 \mathrm{mg} / \mathrm{dL}(<1.8 \mathrm{mmol} / \mathrm{L})$ for adults with $\mathrm{CHD}$ or diabetes, and $<135 \mathrm{mg} / \mathrm{dL}(<3.5 \mathrm{mmol} / \mathrm{L})$ for children. ${ }^{3,9}$ To this aim, several cholesterol-lowering drugs are available; statins are the first choice in adults but also in children, in whom only those proven to be relatively safe should be used. ${ }^{3,10,11}$ Statins effectively reduce $\mathrm{CHD}$ risk in $\mathrm{FH}$ subjects, ${ }^{12}$ however, increasing the dose of statins did not result in further LDL-C level reduction due to a plateau effect, ${ }^{13,14}$ which supports the need for drugs with a complementary mechanism of action to be used in combination with statins to achieve recommended LDL-C targets. ${ }^{3,15}$ These drugs include ezetimibe, mipomersen and lomitapide, or PCSK9 inhibitors. ${ }^{16}$ In addition, some patients are statin-intolerant, and this has a special relevance in $\mathrm{FH}$ patients who need high doses of statins to achieve the recommended LDL-C level target. Mipomersen and lomitapide are registered for patients with homozygous familial hypercholesterolemia $(\mathrm{HoFH})$, while patients with $\mathrm{HeFH}$ will benefit from the combination statins/ezetimibe, which yields an additional reduction of $15 \%-18 \%{ }^{17,18}$ and, more recently, from the monoclonal antibodies directed against PCSK9.

\section{PCSK9 biology and strategies to inhibit PCSK9}

PCSK9 is a serine protease that binds the LDLR and promotes its lysosomal degradation ${ }^{19}$. Through this posttranscriptional mechanism, PCSK9 limits LDL catabolism and increases plasma LDL-C levels. ${ }^{20}$ PCSK9 also targets lipoprotein synthesis in the intestine ${ }^{20}$ further contributing to a disturbed plasma lipid profile. Thus, patients homozygous for gain-of-function mutations in PCSK9 gene present the clinical phenotype of $\mathrm{FH}$ with tendon xanthomas, history of CHD, early myocardial infarction, and stroke. On the contrary, subjects with loss-of-function mutations in PCSK9 gene present with lower plasma LDL-C levels and are protected from coronary artery diseases. ${ }^{21-23}$ Of note, PCSK9 plasma levels predict cardiovascular events in statin-treated patients with well-controlled LDL levels and documented stable coronary artery disease, ${ }^{24}$ further linking PCSK9 to cardiovascular outcomes.
PCSK9 production is mainly regulated by changes in cholesterol levels in the liver via the modulation of the nuclear translocation of the sterol-responsive element-binding protein 2 transcription factor. ${ }^{25,26}$ Once secreted, mature PCSK9 protein undergoes post-translational modifications that can modulate its function, including the cleavage to a truncated protein of about $60 \mathrm{kDa}$ by furin or PC5/6A, 2 members of the proprotein convertase family.

More importantly, PCSK9 plasma levels increase following cholesterol-lowering treatments, a finding observed not only with statins but also with ezetimibe. ${ }^{27-29}$ This mechanism contributes to limiting the pharmacological efficacy of statins and other lipid-lowering strategies as well as provides a mechanisms for understanding the poor correlation between PCSK9 and LDL in circulation. ${ }^{28,29}$ Therefore, given the role of PCSK9 as chaperone in directing the LDLR toward degradation, ${ }^{30}$ the possibility of inhibiting PCSK 9 represents a key approach to enhance the lipid-lowering effect of conventional agents. ${ }^{30}$ From a pharmacological perspective, PCSK 9 could be targeted at different levels from the gene transcription (small interfering RNAs, antisense oligonucleotides) to the circulating protein (anti-PCSK9 monoclonal antibodies or PCSK9 vaccine). ${ }^{30}$

\section{PCSK9 gene silencing}

Gene-silencing approaches are under clinical development, and the results from the first Phase II study, ORION-1, with a siRNA designed to target PCSK9 (inclisiran) were recently published. ${ }^{31} \mathrm{~A}$ single injection of the drug results in LDL-C reduction up to $-36 \%$ while the injection of 2 doses (days 0 and 90) yielded up to $-47.2 \%$ LDL-C reduction after 240 days.

\section{Anti-PCSK 9 antibodies}

Monoclonal antibodies targeted against circulating PCSK9 have been approved for the treatment of patients at very high cardiovascular risk, patients with $\mathrm{FH}$ and statin intolerance.

Three antibodies targeting PCSK9 have been developed (evolocumab, alirocumab, bococizumab) and one, LY3015014 is under development. ${ }^{32}$ Evolocumab and alirocumab are commercially available while the development of bococizumab has been halted as a reduction in the efficacy was observed as a consequence of the induction of neutralizing antibodies. ${ }^{33}$ Results from Phase II studies indicated that PCSK9 therapy reduces LDL-C cholesterol up to $60 \%-70 \%,{ }^{16}$ setting the stage for further evaluating the benefit of anti-PCSK9 monoclonal antibodies in larger cohorts. Anti-PCSK9 therapies not only reduce LDL-C levels 
but also favor atherosclerotic plaque regression, as evidenced by the GLAGOV trial, where in patients with angiographic coronary disease treated with statins, addition of evolocumab resulted in a $-0.95 \%$ decrease in percent atheroma volume after 76 weeks of treatment. ${ }^{34}$ More recently, the FOURIER trial demonstrated that the inhibition of PCSK9 with evolocumab, on a background of statin therapy, reduced the risk of cardiovascular events (median duration of follow-up 26 months) further supporting the benefit of this pharmacological strategy. ${ }^{35}$ The clinical trial for cardiovascular outcomes with the other approved antibody, alirocumab, is ongoing and the results are expected to be released in early 2018 (ODYSSEY OUTCOMES). Of note, results on cardiovascular benefits are also available for bococizumab, which, in high-risk patients (SPIRE-2 trial), resulted in a significant reduction of major cardiovascular events, an effect which could not be detected in the trial involving lower risk patients (SPIRE-1 trial). ${ }^{36}$

These data further reinforce the efficacy of lowering LDL-C by inhibiting PCSK9 and point to the investigation of the efficacy in patients with $\mathrm{HeFH}$.

\section{Clinical trials supporting the use of PCSK9 inhibitors in $\mathrm{HeFH}$}

Phase I-III clinical trials clearly showed the benefit of PCSK9 inhibitors, both alirocumab and evolocumab in patients with $\mathrm{HeFH}$ (Table 2). Data on HeFH patients are available for both.
In the first Phase I trial, alirocumab was tested in 21 $\mathrm{HeFH}$ patients on atorvastatin therapy and LDL-C $>100$ $\mathrm{mg} / \mathrm{dL} .{ }^{37}$ The average reduction of LDL-C levels was $-41 \%$ at the dose of $50 \mathrm{mg}$ and up to $-55 \%$ at the dose of 150 $\mathrm{mg}$. In the 5 patients receiving $150 \mathrm{mg}$ of alirocumab, the degree of LDL-C reduction observed, ranged from -35\% to $\sim-75 \%$. No serious adverse events were reported, with no subjects showing increase in alanine transferase or aspartate transferase levels and $13 \%$ of subjects taking also atorvastatin reported an elevation of creatine kinase 3-time higher the upper limit of the normal range.

In a larger multicenter, randomized, placebo-controlled, Phase II trial, alirocumab was assessed in $77 \mathrm{HeFH}$ patients receiving statins with or without ezetimibe and with LDL-C $>100 \mathrm{mg} / \mathrm{dL} .{ }^{38}$ Patients were randomized to alirocumab 150 , 200 , or $300 \mathrm{mg}$ every 4 weeks, $150 \mathrm{mg}$ every 2 weeks, or placebo every 2 weeks, and were stratified by concomitant use of ezetimibe at baseline. At week 12, LDL-C reductions from baseline to week 12 ranged from 29\% (150 mg every 4 weeks) to $68 \%$ for alirocumab (150 mg every 2 weeks), compared with $\sim-11 \%$ for placebo. Adverse event profiles were similar for alirocumab and placebo; the most common adverse event was injection-site reaction with one patient in the group of alirocumab $300 \mathrm{mg}$ terminating the treatment.

Among Phase III trials with alirocumab, HeFH patients were investigated in the ODYSSEY FH I and FH II, ${ }^{39}$ ODYSSEY JAPAN, ${ }^{40}$ ODYSSEY HIGH $\mathrm{FH}^{41}$ and ODYSSEY

Table 2 Effects of PCSK9 inhibitors evolocumab and alirocumab in $\mathrm{HeFH}$

\begin{tabular}{|c|c|c|c|}
\hline Clinical trial & Subjects (background LLT) & $\begin{array}{l}\text { Intervention (dose, } \\
\text { frequency) }\end{array}$ & $\begin{array}{l}\text { LDL-C (\% change } \\
\text { from baseline) }\end{array}$ \\
\hline \multicolumn{4}{|l|}{ EVOLOCUMAB } \\
\hline $\begin{array}{l}\text { RUTHERFORD }{ }^{45} \\
12 \text { weeks }\end{array}$ & $\begin{array}{l}\mathrm{HeFH}, \mathrm{LDL}-\mathrm{C} \geq 100 \mathrm{mg} / \mathrm{dL} \\
\text { (statin } \pm \text { ezetimibe) }\end{array}$ & $420 \mathrm{mg}$ Q4W & $-56.4 \%$ \\
\hline $\begin{array}{l}\text { RUTHERFORD- } 2^{46} \\
12 \text { weeks }\end{array}$ & $\begin{array}{l}\mathrm{HeFH}, \mathrm{LDL}-\mathrm{C} \geq 100 \mathrm{mg} / \mathrm{dL} \\
\text { (statin } \pm \text { ezetimibe) }\end{array}$ & $\begin{array}{l}140 \mathrm{mg} \mathrm{Q2W} \\
420 \mathrm{mg} \text { Q4W }\end{array}$ & $\begin{array}{l}-59.2 \% \\
-61.3 \%\end{array}$ \\
\hline \multicolumn{4}{|l|}{ ALIROCUMAB } \\
\hline $\begin{array}{l}\text { ODYSSEY FH I and FH II } \\
78 \text { weeks }\end{array}$ & $\begin{array}{l}\mathrm{HeFH}, \\
\mathrm{LDL}-\mathrm{C} \geq 100 \mathrm{mg} / \mathrm{dL} \text { (for primary prevention) or LDL-C } \\
\geq 70 \mathrm{mg} / \mathrm{dL} \text { (for secondary prevention) } \\
\text { (max tolerated statin } \pm \text { other } \mathrm{LLT} \text { ) }\end{array}$ & $\begin{array}{l}75 \mathrm{mg} \text { Q2W (increased at } 150 \mathrm{mg} \\
\text { Q2W if LDL-C } \geq 70 \mathrm{mg} / \mathrm{dL} \text { at } \\
\text { week 8) }\end{array}$ & $\begin{array}{l}\text { FH I: }-57.9 \% \\
\text { FH II: }-51.4 \%\end{array}$ \\
\hline $\begin{array}{l}\text { ODYSSEY HIGH FH }{ }^{41} \\
78 \text { weeks }\end{array}$ & $\begin{array}{l}\text { HeFH, LDL-C } \geq 160 \\
\text { (stable LLT) }\end{array}$ & $150 \mathrm{mg}$ Q2W & $-39 \%$ \\
\hline $\begin{array}{l}\text { ODYSSEY ESCAPE }{ }^{42} \\
18 \text { weeks }\end{array}$ & $\begin{array}{l}\text { HeFH undergoing lipoprotein apheresis } \\
\text { (lipoprotein apheresis + stable LLT + diet + exercise) }\end{array}$ & $150 \mathrm{mg}$ Q2W & $-42.5 \%$ \\
\hline $\begin{array}{l}\text { ODYSSEY LONG TERM }{ }^{43} \\
78 \text { weeks }\end{array}$ & $\begin{array}{l}\text { HeFH or established } \mathrm{CHD} \text { or CHD equivalent, } \\
\mathrm{LDL}-\mathrm{C} \geq 70 \mathrm{mg} / \mathrm{dL} \\
\text { (max tolerated statin } \pm \text { other LLT) }\end{array}$ & $150 \mathrm{mg}$ Q2w & $-61.9 \%$ \\
\hline $\begin{array}{l}\text { ODYSSEY OLE } \\
\text { I76 weeks }\end{array}$ & $\begin{array}{l}\text { HeFH who have completed one of the } 4 \text { parent studies } \\
\text { (max tolerated statin } \pm \text { other LLT) }\end{array}$ & ONGOING & \\
\hline
\end{tabular}

Abbreviations: CHD, coronary heart disease; FH, familial hypercholesterolemia; HeFH, heterozygous familial hypercholesterolemia; LDL-C, low-density lipoprotein cholesterol; LLT, lipid-lowering therapy; PCSK9, proprotein convertase subtilisin kexin 9; Q4W, every 4 weeks; Q2W, every 2 weeks. 
ESCAPE. ${ }^{42}$ In ODYSSEY FH I and FH II 735 patients with $\mathrm{HeFH}$ receiving maximum tolerated statin doses with or without other lipid-lowering therapy were enrolled..$^{39}$ Patients were randomized to alirocumab $75 \mathrm{mg}$ every 2 weeks (with possible increase to $150 \mathrm{mg}$ at week 12, if LDL-C goal was not reached at week 8), or placebo. At week 24, mean reductions in LDL-C compared with placebo were $58 \%$ in FH I and 51\% in FH II. Safety and tolerability were generally comparable in the alirocumab and placebo groups. The ODYSSEY JAPAN study included $41 \mathrm{HeFH}$ patients and alirocumab treatment resulted in LDL-C reduction similar to what was observed with the other studies. ${ }^{40}$ The ODYSSEY HIGH FH trial included 107 patients with severe $\mathrm{HeFH}$, (LDL-C levels $>160 \mathrm{mg} / \mathrm{dL}$ on maximally tolerated statins with or without other lipid-lowering therapies). ${ }^{40}$ Patients were randomized to placebo or alirocumab $150 \mathrm{mg}$ every 2 weeks. At week 24, the relative reduction observed in LDL-C was $-45.7 \%$ compared with $-6.6 \%$ in the placebo group. As seen in other trials, alirocumab was generally well tolerated and adverse event rates were generally similar to those of the placebo group. The ODYSSEY ESCAPE trial evaluated the effect of 12-week treatment with alirocumab in $\mathrm{HeFH}$ patients undergoing regular lipoprotein apheresis every 1 or 2 weeks. ${ }^{42}$ More than $90 \%$ patients on alirocumab at least halved the standardized rate of apheresis $(-53.7 \%$ change from baseline at week 6 ), compared with $+1.6 \%$ in the placebo group, ${ }^{42}$ and $63.4 \%$ of patients on alirocumab had not apheresis procedures during 12 weeks. ${ }^{42}$ In contrast to apheresis procedures, characterized by a rapid increase of LDL-C levels toward pre-apheresis levels, alirocumab maintained LDL-C levels persistently low, ${ }^{42}$ which may translate into a clinical benefit over the long term.

In agreement with this last observation, available data indicate that the effect of alirocumab persists over time and indeed after 78 weeks of treatment, the results of the ODYSSEY LONG TERM study ${ }^{43}$ showed that LDL-C levels were still significantly lower compared with what was observed in the placebo group; of note, this study included 415 patients with $\mathrm{HeFH}$ where the benefit observed was similar to that of the entire cohort and in line with what was reported for the other studies in $\mathrm{HeFH}$ patients. ${ }^{44}$

A pooled analysis of the safety data for alirocumab studies in $\mathrm{HeFH}$ patients of treatment was recently published. Data are available for more than $800 \mathrm{HeFH}$ patients treated with alirocumab versus 418 with placebo (both groups were on the maximal tolerated dose of statins + other lipid-lowering therapies) up to 78 weeks. Regardless of the alirocumab dose, rates of treatment-emergent adverse events (TEAEs) were similar in alirocumab (80.5\%) and placebo-treated patients $(83.0 \%)$. TEAEs reported in $\geq 2 \%$ of patients receiving alirocumab were generally comparable with the placebo group, except for a higher proportion of patients experiencing injection-site reactions in the alirocumab group (11.4\%) compared with placebo $(8.6 \%){ }^{44}$

The efficacy and safety of evolocumab in HeFH patients were also tested in 2 studies; the RUTHERFORD study was a Phase II trial designed to evaluate the effect of evolocumab 350 or $420 \mathrm{mg}$ versus placebo every 4 weeks in 111 patients with clinical diagnosis of FH (SB criteria) and LDL-C levels above $100 \mathrm{mg} / \mathrm{dL}$ despite statin therapy with or without ezetimibe. ${ }^{45}$ After 12 weeks of treatment, LDL-C was reduced by $\sim 55 \%$ with evolocumab $420 \mathrm{mg}$, compared with a $1 \%$ increase with placebo $(P<0.001)$.

Four patients in the evolocumab $350 \mathrm{mg}$ group and one patient in the $420 \mathrm{mg}$ group were considered poor responders based on a $<15 \%$ reduction in LDL-C at week $12 .{ }^{45}$ The 3 most common treatment-related adverse events were injection-site pain (reported in 3.6\% of evolocumab-treated patients), headache $(1.8 \%)$, and skin burning sensation $(3.6 \%){ }^{45}$

Evolocumab was further investigated in the Phase III RUTHERFORD-2 trial, in which 331 patients with $\mathrm{HeFH}$ were randomly assigned to evolocumab $140 \mathrm{mg}$ twice a month, $420 \mathrm{mg}$ monthly, placebo twice a month, or placebo monthly. ${ }^{46}$ After 12 weeks, evolocumab was associated with LDL-C reductions of $\sim 60 \%$ compared with the placebo group. ${ }^{46}$ The rate of adverse events was similar between the evolocumab and placebo groups and overall evolocumab was well tolerated. ${ }^{46}$

As described above, patients who will benefit from antiPCSK9 therapy are, without any doubt, those with $\mathrm{HeFH}$ while for those with $\mathrm{HoFH}$, the benefit will be linked to the mutation underlying the disease. Indeed, based on the mechanism of action (a robust induction of LDLR-mediated clearance of LDL), the benefit of anti-PCSK9 therapy should be more marked in $\mathrm{FH}$ patients who retain a partial functionality of the LDLR (LDLR defective), while it would be less effective in those with a severe mutation of the LDLR (LDLR null) or those with other mutations impacting the LDLR axis (such as LDLRAP1). On the contrary, it is expected that the drug would be highly effective in patients with gain of function mutations of PCSK9.

For the treatment of $\mathrm{HoFH}$, the TESLA clinical trials have specifically addressed the benefit of evolocumab in these patients. ${ }^{47,48}$ A similar observation is available also for alirocumab on HoFH patients, although in a limited cohort: ali- 
rocumab normalizes LDLR expression in receptor-defective HoFH fibroblasts, while no effect is observed in receptornegative HoFH. ${ }^{49}$ An interim subset analysis of the TAUSSIG trial confirms the robust and durable reductions in LDL-C levels obtained with evolocumab in HoFH patients; in addition, it provides positive results for subjects receiving apheresis in addition to stable lipid-lowering therapy and, even more important, for adolescent patients. ${ }^{50}$ The data indicate a reduction of LDL-C levels on the top of available therapies of approximately $-26 \%$. Although relevant, other drugs with different mechanisms of action, such as lomitapide, have produced a greater magnitude of reduction in $\mathrm{HoFH}$ patients ${ }^{51}$ while for mipomersen, the reduction in LDL-C achieved is closer to that observed for PCSK9 inhibitors. ${ }^{52}$

\section{Conclusions and future directions}

Available data suggest that $\mathrm{HeFH}$ patients would benefit most from additional lipid lowering with an anti-PCSK9 antibody. Data on cardiovascular benefit of evolocumab have been recently published (FOURIER trial), ${ }^{35}$ reporting data on overall and cardiovascular mortality as well as the incidence of myocardial infarction. The study included patients with atherosclerotic cardiovascular disease and LDL-C $\geq 70 \mathrm{mg} / \mathrm{dL}$ who were receiving statin therapy. ${ }^{35} \mathrm{~A}$ sub-analysis of cardiovascular outcome in FH patients enrolled in the study is not yet available but it is reasonable to believe that the drugs will be as effective in this cohort. However, given the mechanism of action of anti-PCSK9, it should be taken into account that some HeFH patients with null mutation on the LDL receptor will probably experience a minor benefit following monoclonal antibody therapy, although the RUTHERFORD-2 study did not observe different LDL-C lowering response to evolocumab in patients with defective or negative LDLR ${ }^{46}$ All the studies of PCSK9 inhibition in HeFH subjects, in addition, reported a good tolerability and rate of adverse events were similar between the evolocumab/alirocumab and placebo groups (Table 3 ), as also reported by a recent pooled analysis of studies with alirocumab in $\mathrm{HeFH}$ patients. ${ }^{44}$

PCSK9 inhibition was proven to also reduce the levels of lipoprotein(a) (Lp(a)), an LDL-like particle, which represents a cardiovascular risk factor independently of LDL-C levels. ${ }^{53}$ Indeed FH patients, especially those with cardiovascular disease, exhibit significantly higher plasma levels of $\mathrm{Lp}(\mathrm{a})$ compared with their non-affected relatives. ${ }^{54} \mathrm{Lp}(\mathrm{a})$ was proposed to play causal role in aortic valve calcification development in asymptomatic statin-treated HeFH. ${ }^{55}$ These observations support the need to asses $\mathrm{Lp}$ (a) levels in FH patients to identify those who could benefit from more aggressive lipid-lowering treatments.
Table 3 TEAE frequency in PCSK9 mAbs clinical trials in $\mathrm{HeFH}$ patients

\begin{tabular}{lll}
\hline TEAEs & PCSK9 mAbs & Placebo \\
\hline TEAEs (any) & $51 \%-81.7 \%$ & $28 \%-82.5 \%$ \\
Serious adverse events & $1.8 \%-18.7 \%$ & $0 \%-19.5 \%$ \\
Leading to death & $0 \%-1.9 \%$ & $0 \%-1.3 \%$ \\
Leading to treatment discontinuation & $0 \%-7.2 \%$ & $0 \%-6.1 \%$ \\
Common adverse events & & \\
Injection site reaction & $2.4 \%-12.4 \%$ & $0 \%-11 \%$ \\
$\quad$ Nasopharyngitis & $7 \%-13.9 \%$ & $4 \%-22.2 \%$ \\
$\quad$ Influenza & $4.5 \%-14.4 \%$ & $2.9 \%-10.7 \%$ \\
$\quad$ Headache & $4 \%-9.6 \%$ & $0 \%-8.9 \%$ \\
$\quad$ Diarrhea & $1.8 \%-9.8 \%$ & $0 \%-8.6 \%$ \\
Nausea & $1.8 \%-4.9 \%$ & $0 \%-14.3 \%$ \\
Myalgia & $1.9 \%-9.8 \%$ & $0 \%-8.6 \%$ \\
\hline
\end{tabular}

Abbreviations: $\mathrm{HeFH}$, heterozygous familial hypercholesterolemia; $\mathrm{mAb}$, monoclonal antibody; PCSK9, proprotein convertase subtilisin kexin 9; TEAEs, treatment-emergent adverse events.

In contrast to statins, PCSK9 mAbs reduce $\mathrm{Lp}$ (a) levels by up to $30 \%,{ }^{56,57}$ but the exact mechanism by which such reduction takes place is still unclear. LDL-C and Lp(a) levels decrease concomitantly during therapy with PCSK9 mAbs, and patients achieving lower LDL-C levels also show larger $\mathrm{Lp}$ (a) reductions. ${ }^{58}$ Based on these observations, the effect on $\mathrm{Lp}$ (a) levels in $\mathrm{HeFH}$ patients treated with evolocumab or alirocumab was evaluated. The RUTHERFORD studies reported reductions ranging from $-23 \%$ up to $-31.6 \%$ versus placebo; ${ }^{45,46}$ similar reductions were observed in the studies of the ODYSSEY program performed in FH subjects $(-14.8 \%$ to $-25.6 \%$ versus placebo), ${ }^{39,41,43}$ with the exception of the ODYSSEY ESCAPE trial that reported a $-4.1 \%$ Lp(a) level reduction versus placebo. ${ }^{42}$ Also HoFH showed significant $\mathrm{Lp}$ (a) level reductions, apparently independent of the genetic defect. ${ }^{47,48}$ The clinical relevance of $\mathrm{Lp}$ (a) reduction remains to be addressed, however.

Do the achieved very low LDL-C levels pose problems of safety? Recently, an analysis of pooled data from 14 trials on alirocumab indicated that LDL-C levels $<25$ or $<15 \mathrm{mg} / \mathrm{dL}$ were not associated with a higher incidence of treatment-emergent event rates or neurocognitive events, ${ }^{59}$ although the incidence of cataract appeared to be increased in subjects achieving LDL-C levels $<25 \mathrm{mg} / \mathrm{dL}$. ${ }^{59}$ This finding, however, might be the consequence of the comparison of non-randomized subgroups. Due to the need of lifelong therapy in patients with $\mathrm{FH}$, the safety of long-term exposure to pharmacologically induced very low levels of LDL-C remains to be determined.

A final aspect to be considered is the cost-effectiveness of anti-PCSK9 therapy in $\mathrm{HeFH}$ patients. To date, an analysis is available for the US market. ${ }^{60}$ Adding PCSK9 inhibitors to statins in $\mathrm{HeFH}$ was estimated to prevent 316,300 major adverse cardiovascular events at a cost of 
\$503.000 per quality-adjusted life year (QALY) gained compared with adding ezetimibe to statins. ${ }^{60}$ Reaching the desired QALY threshold of $\$ 100.000$ will need an important reduction of annual drug price. However, a more precise determination of the cost-effectiveness of PCSK9 therapy can only be performed once long-term data on clinical outcomes on $\mathrm{HeFH}$ patients for PCSK9 inhibitors becomes available.

\section{Acknowledgments}

The work of the authors is supported by: Fondazione Cariplo 2015-0524 and 2015-0564 (ALC), and 2016-0852 (GDN); H2020 REPROGRAM PHC-03-2015/667837-2 (ALC); Telethon Foundation (GGP13002) (GDN), Ministero della Salute GR-2011-02346974 (GDN); Aspire Cardiovascular Grant 2016-WI218287 (GDN).

\section{Disclosure}

The authors have received research funding, and/or honoraria for advisory boards, consultancy or speaker bureau from Aegerion (ALC, GDN), Amgen (ALC, GDN), AstraZeneca (ALC), Eli Lilly (ALC), Genzyme (ALC), Mediolanum (ALC), Merck or MSD (ALC), Pfizer (ALC, GDN), Recordati (ALC, GDN), Rottapharm (ALC), Sanofi-Regeneron (ALC, GDN), Sigma-Tau (ALC). AP reports no conflicts of interest in this work.

\section{References}

1. Hopkins PN, Toth PP, Ballantyne CM, Rader DJ; National Lipid Association Expert Panel on Familial Hypercholesterolemia. Familial hypercholesterolemias: prevalence, genetics, diagnosis and screening recommendations from the National Lipid Association Expert Panel on Familial Hypercholesterolemia. J Clin Lipidol. 2011;5 (Suppl 3): S9-S17.

2. Sharifi M, Futema M, Nair D, Humphries SE. Genetic architecture of familial hypercholesterolaemia. Curr Cardiol Rep. 2017;19(5):44.

3. Nordestgaard BG, Chapman MJ, Humphries SE, et al; European Atherosclerosis Society Consensus Panel. Familial hypercholesterolaemia is underdiagnosed and undertreated in the general population: guidance for clinicians to prevent coronary heart disease: consensus statement of the European Atherosclerosis Society. Eur Heart J. 2013;34(45):3478-3490;.

4. De Backer G, Besseling J, Chapman J, et al; EUROASPIRE Investigators. Prevalence and management of familial hypercholesterolaemia in coronary patients: an analysis of EUROASPIRE IV, a study of the European Society of Cardiology. Atherosclerosis. 2015;241(1):169-175.

5. Cuchel M, Bruckert E, Ginsberg HN, et al; European Atherosclerosis Society Consensus Panel on Familial Hypercholesterolaemia. Homozygous familial hypercholesterolaemia: new insights and guidance for clinicians to improve detection and clinical management. A position paper from the Consensus Panel on Familial Hypercholesterolaemia of the European Atherosclerosis Society. Eur Heart J. 2014;35(32):2146-2157.

6. Defesche JC, Lansberg PJ, Umans-Eckenhausen MA, Kastelein JJ. Advanced method for the identification of patients with inherited hypercholesterolemia. Semin Vasc Med. 2004;4(1):59-65.
7. Williams RR, Hunt SC, Schumacher MC, et al. Diagnosing heterozygous familial hypercholesterolemia using new practical criteria validated by molecular genetics. Am J Cardiol. 1993;72(2):171-176.

8. Risk of fatal coronary heart disease in familial hypercholesterolaemia. Scientific Steering Committee on behalf of the Simon Broome Register Group. BMJ. 1991;303(6807):893-896.

9. Catapano AL, Graham I, De Backer G, et al; Authors/Task Force Members; Additional Contributor. 2016 ESC/EAS Guidelines for the Management of Dyslipidaemias. Eur Heart J. 2016;37(39):2999-3058.

10. McCrindle BW, Ose L, Marais AD. Efficacy and safety of atorvastatin in children and adolescents with familial hypercholesterolemia or severe hyperlipidemia: a multicenter, randomized, placebo-controlled trial. $J$ Pediatr. 2003;143(1):74-80.

11. Wiegman A, Hutten BA, de Groot E, et al. Efficacy and safety of statin therapy in children with familial hypercholesterolemia: a randomized controlled trial. JAMA. 2004;292(3):331-337.

12. Versmissen J, Oosterveer DM, Yazdanpanah M, et al. Efficacy of statins in familial hypercholesterolaemia: a long term cohort study. BMJ. 2008;337:a2423.

13. Raal FJ, Pappu AS, Illingworth DR, et al. Inhibition of cholesterol synthesis by atorvastatin in homozygous familial hypercholesterolaemia. Atherosclerosis. 2000;150(2):421-428.

14. Hagemenas FC, Pappu AS, Illingworth DR. The effects of simvastatin on plasma lipoproteins and cholesterol homeostasis in patients with heterozygous familial hypercholesterolaemia. Eur J Clin Invest. 1990;20(2):150-157.

15. Hovingh GK, Davidson MH, Kastelein JJ, O'Connor AM. Diagnosis and treatment of familial hypercholesterolaemia. Eur Heart J. 2013;34(13):962-971.

16. Norata GD, Ballantyne CM, Catapano AL. New therapeutic principles in dyslipidaemia: focus on LDL and Lp(a) lowering drugs. Eur Heart J. 2013;34(24):1783-1789.

17. Pisciotta L, Fasano T, Bellocchio A, et al. Effect of ezetimibe coadministered with statins in genotype-confirmed heterozygous $\mathrm{FH}$ patients Atherosclerosis. 2007;194(2): e116-e122.

18. Kastelein JJ, Akdim F, Stroes ES, et al. Simvastatin with or without ezetimibe in familial hypercholesterolemia. $N$ Engl J Med. 2008;358(14):1431-1443.

19. Lagace TA. PCSK 9 and LDLR degradation: regulatory mechanisms in circulation and in cells. Curr Opin Lipidol. 2014;25(5):387-393.

20. Norata GD, Tavori H, Pirillo A, Fazio S, Catapano AL. Biology of proprotein convertase subtilisin kexin 9: beyond low-density lipoprotein cholesterol lowering. Cardiovasc Res. 2016;112(1):429-442.

21. Cohen JC, Boerwinkle E, Mosley TH Jr, Hobbs HH. Sequence variations in PCSK9, low LDL, and protection against coronary heart disease. $N$ Engl J Med. 2006;354(12):1264-1272.

22. Cohen J, Pertsemlidis A, Kotowski IK, Graham R, Garcia CK, Hobbs HH. Low LDL cholesterol in individuals of African descent resulting from frequent nonsense mutations in PCSK9. Nat Genet. 2005;37(2):161-165.

23. Kathiresan S. A PCSK 9 missense variant associated with a reduced risk of early-onset myocardial infarction. $N$ Engl J Med. 2008;358(21):2299-2300.

24. Werner C, Hoffmann MM, Winkler K, Bohm M, Laufs U. Risk prediction with proprotein convertase subtilisin/kexin type 9 (PCSK9) in patients with stable coronary disease on statin treatment. Vascul Pharmacol. 2014;62(2):94-102.

25. Jeong HJ, Lee HS, Kim KS, Kim YK, Yoon D, Park SW. Steroldependent regulation of proprotein convertase subtilisin/kexin type 9 expression by sterol-regulatory element binding protein-2. J Lipid Res. 2008;49(2):399-409.

26. Norata GD, Tibolla G, Catapano AL. PCSK9 inhibition for the treatment of hypercholesterolemia: promises and emerging challenges. Vascul Pharmacol. 2014;62(2):103-111.

27. Careskey HE, Davis RA, Alborn WE, Troutt JS, Cao G, Konrad RJ. Atorvastatin increases human serum levels of proprotein convertase subtilisin/kexin type 9. J Lipid Res. 2008;49(2):394-398. 
28. Awan Z, Seidah NG, MacFadyen JG, et al. Rosuvastatin, proprotein convertase subtilisin/kexin type 9 concentrations, and LDL cholesterol response: the JUPITER trial. Clin Chem. 2012;58(1):183-189.

29. Welder G, Zineh I, Pacanowski MA, Troutt JS, Cao G, Konrad RJ. High-dose atorvastatin causes a rapid sustained increase in human serum PCSK 9 and disrupts its correlation with LDL cholesterol. J Lipid Res. 2010;51(9):2714-2721.

30. Tibolla G, Norata GD, Artali R, Meneghetti F, Catapano AL. Proprotein convertase subtilisin/kexin type 9 (PCSK9): from structurefunction relation to therapeutic inhibition. Nutr Metab Cardiovasc Dis. 2011;21(11):835-843.

31. Ray KK, Landmesser U, Leiter LA, et al. Inclisiran in patients at high cardiovascular risk with elevated LDL cholesterol. $N$ Engl J Med. 2017;376(15):1430-1440.

32. Kastelein JJ, Nissen SE, Rader DJ, et al. Safety and efficacy of LY30,15,014, a monoclonal antibody to proprotein convertase subtilisin/ kexin type 9 (PCSK9): a randomized, placebo-controlled Phase 2 study. Eur Heart J. 2016;37(17):1360-1369.

33. Ridker PM, Tardif JC, Amarenco P, et al; SPIRE Investigators. Lipidreduction variability and antidrug-antibody formation with bococizumab. N Engl J Med. 2017;376(16):1517-1526.

34. Nicholls SJ, Puri R, Anderson T, et al. Effect of evolocumab on progression of coronary disease in statin-treated patients: the GLAGOV randomized clinical trial. JAMA. 2016;316(22):2373-2384.

35. Sabatine MS, Giugliano RP, Keech AC, et al; FOURIER Steering Committee and Investigators. Evolocumab and clinical outcomes in patients with cardiovascular disease. $N$ Engl J Med. 2017;376(18): 1713-1722.

36. Ridker PM, Revkin J, Amarenco P, et al; SPIRE Cardiovascular Outcome Investigators. Cardiovascular efficacy and safety of bococizumab in high-risk patients. $N$ Engl J Med. 2017;376(16):1527-1539.

37. Stein EA, Mellis S, Yancopoulos GD, et al. Effect of a monoclonal antibody to PCSK9 on LDL cholesterol. N Engl J Med. 2012;366(12): 1108-1118.

38. Stein EA, Gipe D, Bergeron J, et al. Effect of a monoclonal antibody to PCSK9, REGN727/SAR2,36,553, to reduce low-density lipoprotein cholesterol in patients with heterozygous familial hypercholesterolaemia on stable statin dose with or without ezetimibe therapy: a phase 2 randomised controlled trial. Lancet. 2012;380(9836):29-36.

39. Kastelein JJ, Ginsberg HN, Langslet G, et al. ODYSSEY FH I and FH II: 78 week results with alirocumab treatment in 735 patients with heterozygous familial hypercholesterolaemia. Eur Heart J. 2015;36(43):2996-3003.

40. Teramoto T, Kobayashi M, Tasaki H, et al. Efficacy and Safety of Alirocumab in Japanese Patients With Heterozygous Familial Hypercholesterolemia or at High Cardiovascular Risk With Hypercholesterolemia Not Adequately Controlled With Statins- ODYSSEY JAPAN Randomized Controlled Trial. Circ J. 2016;80(9):1980-1987.

41. Ginsberg HN, Rader DJ, Raal FJ, et al. Efficacy and Safety of Alirocumab in Patients with Heterozygous Familial Hypercholesterolemia and LDL-C of $160 \mathrm{mg} / \mathrm{dl}$ or Higher. Cardiovasc Drugs Ther. 2016;30(5):473-483.

42. Moriarty PM, Parhofer KG, Babirak SP, et al. Alirocumab in patients with heterozygous familial hypercholesterolaemia undergoing lipoprotein apheresis: the ODYSSEY ESCAPE trial. Eur Heart J. 2016;37(48):3588-3595.

43. Robinson JG, Farnier M, Krempf M, et al; ODYSSEY LONG TERM Investigators. Efficacy and safety of alirocumab in reducing lipids and cardiovascular events. $N$ Engl J Med. 2015;372(16):1489-1499.

44. Kastelein JJ, Hovingh GK, Langslet G, et al. Efficacy and safety of the proprotein convertase subtilisin/kexin type 9 monoclonal antibody alirocumab vs placebo in patients with heterozygous familial hypercholesterolemia. J Clin Lipidol. 2017;11(1):195-203.e4.
45. Raal F, Scott R, Somaratne R, et al. Low-density lipoprotein cholesterollowering effects of AMG 145, a monoclonal antibody to proprotein convertase subtilisin/kexin type 9 serine protease in patients with heterozygous familial hypercholesterolemia: the Reduction of LDL-C with PCSK9 Inhibition in Heterozygous Familial Hypercholesterolemia Disorder (RUTHERFORD) randomized trial. Circulation. 2012;126(20):2408-2417.

46. Raal FJ, Stein EA, Dufour R, et al; RUTHERFORD-2 Investigators. PCSK9 inhibition with evolocumab (AMG 145) in heterozygous familial hypercholesterolaemia (RUTHERFORD-2): a randomised, doubleblind, placebo-controlled trial. Lancet. 2015;385(9965):331-340.

47. Stein EA, Honarpour N, Wasserman SM, Xu F, Scott R, Raal FJ. Effect of the proprotein convertase subtilisin/kexin 9 monoclonal antibody, AMG 145, in homozygous familial hypercholesterolemia. Circulation. 2013;128(19):2113-2120.

48. Raal FJ, Honarpour N, Blom DJ, et al; TESLA Investigators. Inhibition of PCSK9 with evolocumab in homozygous familial hypercholesterolaemia (TESLA Part B): a randomised, double-blind, placebo-controlled trial. Lancet. 2015;385(9965):341-350.

49. Lambert G, Chatelais M, Petrides F, et al. Normalization of low-density lipoprotein receptor expression in receptor defective homozygous familial hypercholesterolemia by inhibition of PCSK9 with alirocumab. J Am Coll Cardiol. 2014;64(21):2299-2300.

50. Raal FJ, Hovingh GK, Blom D, et al. Long-term treatment with evolocumab added to conventional drug therapy, with or without apheresis, in patients with homozygous familial hypercholesterolaemia: an interim subset analysis of the open-label TAUSSIG study. Lancet Diabetes Endocrinol. 2017;5(4):280-290.

51. Cuchel M, Meagher EA, du Toit Theron H, et al; Phase $3 \mathrm{HoFH}$ Lomitapide Study investigators. Efficacy and safety of a microsomal triglyceride transfer protein inhibitor in patients with homozygous familial hypercholesterolaemia: a single-arm, open-label, phase 3 study. Lancet. 2013;381(9860):40-46.

52. Raal FJ, Santos RD, Blom DJ, et al. Mipomersen, an apolipoprotein B synthesis inhibitor, for lowering of LDL cholesterol concentrations in patients with homozygous familial hypercholesterolaemia: a randomised, double-blind, placebo-controlled trial. Lancet. 2010;375(9719):998-1006.

53. Nordestgaard BG, Chapman MJ, Ray K, et al; European Atherosclerosis Society Consensus Panel. Lipoprotein(a) as a cardiovascular risk factor: current status. Eur Heart J. 2010;31(23):2844-2853.

54. Alonso R, Andres E, Mata N, et al; SAFEHEART Investigators. Lipoprotein(a) levels in familial hypercholesterolemia: an important predictor of cardiovascular disease independent of the type of LDL receptor mutation. J Am Coll Cardiol. 2014;63(19):1982-1989.

55. Vongpromek R, Bos S, Ten Kate GJ, et al. Lipoprotein(a) levels are associated with aortic valve calcification in asymptomatic patients with familial hypercholesterolaemia. J Intern Med. 2015;278(2):166-173.

56. Raal FJ, Giugliano RP, Sabatine MS, et al. Reduction in lipoprotein(a) with PCSK9 monoclonal antibody evolocumab (AMG 145): a pooled analysis of more than 1,300 patients in 4 phase II trials. $\mathrm{J} \mathrm{Am} \mathrm{Coll}$ Cardiol. 2014;63(13):1278-1288.

57. Gaudet D, Kereiakes DJ, McKenney JM, et al. Effect of alirocumab, a monoclonal proprotein convertase subtilisin/kexin 9 antibody, on lipoprotein(a) concentrations (a pooled analysis of $150 \mathrm{mg}$ every two weeks dosing from phase 2 trials). Am J Cardiol. 2014;114(5):711-715.

58. Raal FJ, Giugliano RP, Sabatine MS, et al. PCSK9 inhibition-mediated reduction in $\mathrm{Lp}(\mathrm{a})$ with evolocumab: an analysis of 10 clinical trials and the LDL receptor's role. J Lipid Res. 2016;57(6):1086-1096.

59. Robinson JG, Rosenson RS, Farnier M, et al. Safety of Very Low LowDensity Lipoprotein Cholesterol Levels With Alirocumab: Pooled Data From Randomized Trials. J Am Coll Cardiol. 2017;69(5):471-482.

60. Kazi DS, Moran AE, Coxson PG, et al. Cost-effectiveness of PCSK9 inhibitor therapy in patients with heterozygous familial hypercholesterolemia or atherosclerotic cardiovascular disease. JAMA. 2016;316(7):743-753. 
Vascular Health and Risk Management is an international, peerreviewed journal of therapeutics and risk management, focusing on concise rapid reporting of clinical studies on the processes involved in the maintenance of vascular health; the monitoring, prevention and treatment of vascular disease and its sequelae; and the involvement of metabolic disorders, particularly diabetes. This journal is indexed on PubMed Central and MedLine. The manuscript management system is completely online and includes a very quick and fair peer-review system, which is all easy to use. Visit http://www.dovepress.com/ testimonials.php to read real quotes from published authors. 